\title{
Tumour necrosis factor- $\alpha$ production in human alveolar macrophages: modulation by inhaled corticosteroid
}

\author{
B.G. Marshall*, A. Wangoo*, L.I. Harrison***, D.B. Young**, R.J. Shaw*
}

Tumour necrosis factor- $\alpha$ production in human alveolar macrophages: modulation by inhaled corticosteroid. B.G. Marshall, A. Wangoo, L.I. Harrison, D.B. Young, R.J. Shaw. (C) ERS Journals Ltd 2000.

ABSTRACT: Using an ex vivo alveolar macrophage model, the hypothesis that inhaled preparations of corticosteroids might have important anti-inflammatory effects on cells of the peripheral airway was tested.

The tumour necrosis factor (TNF)- $\alpha$-inducing potential of three glycolipid preparations from nonpathogenic (arabinofuranasyl lipoarabinomannan (LAM (AraLAM)) and virulent (mannase LAM (ManLAM)) mycobacteria and Gram-negative bacteria (lipopolysaccharide (LPS)), in primary alveolar macrophage preparations was investigated. A novel inhaled chlorofluorocarbon (CFC)-free preparation of beclomethasone dipropionate (hydrofluoroalkane 134a (HFA)-BDP) with increased peripheral lung deposition was investigated for its ability to modulate glycolipidinduced TNF- $\alpha$ production by human alveolar macrophages, in comparison with a CFC-containing preparation and placebo.

Compared to the basal TNF- $\alpha$ bioactivity of $0.72 \mathrm{ng} \cdot \mathrm{mL}^{-1}$ (geometric mean), the TNF- $\alpha$ bioactivity in the macrophage preparation increased following incubation with LPS (138 ng. $\left.\mathrm{mL}^{-1}, \mathrm{p}<0.001\right)$, AraLAM $\left(12.6 \mathrm{ng} \cdot \mathrm{mL}^{-1}, \mathrm{p}<0.001\right)$ and ManLAM $(1.42$ $\left.\mathrm{ng} \cdot \mathrm{mL}^{-1}, \mathrm{p}=0.02\right)$. HFA-BDP, administered in vivo, significantly reduced LPS- and ManLAM-induced TNF- $\alpha$ production by alveolar macrophages cultured ex vivo. No change in glycolipid-induced TNF- $\alpha$ production was observed following in vivo administration of CFC-BDP or HFA-placebo.

This is the first demonstration of an immunomodulatory effect on alveolar cells of corticosteroid delivered via metered dose inhaler. The present findings suggest that alveolar deposition of beclomethasone dipropionate is capable of modulating the inflammatory potential of the alveolar macrophage population.

Eur Respir J 2000; 15: 764-770.
Depts of * Respiratory Medicine, National Heart and Lung Institute, and $* *$ Medical Microbiology, Division of Investigative Science, Imperial College School of Medicine, St. Mary's Campus, London, UK and ***3M Pharmaceuticals, St Paul, MN, USA

Correspondence: B.G. Marshall, Dept of Respiratory Medicine, Southampton University Hospitals NHS Trust, Tremona Road, Southampton, UK. Fax: 4423807 94626

Keywords: Alveolar macrophage

lipoarabinomannan

steroid

tumour necrosis factor

Received: December 91998

Accepted after revision December 91999

This study was supported by an educational grant received from $3 \mathrm{M}$ Pharmaceuticals. B.G. Marshall is supported by the British Medical Association and A. Wangoo by the Wellcome Trust.
Tumour necrosis factor- $\alpha$ (TNF- $\alpha$ ), a key inflammatory mediator, is released from cells of macrophage or monocyte lineage, such as alveolar macrophages. TNF- $\alpha$ has been implicated in the pathogenesis of asthma [1] and interstitial lung diseases [2]. It also plays an important role in both protective [3] and pathological immune responses to Gram-negative bacteria and mycobacteria [4].

The major antigenic lipoglycans of Gram-negative bacteria and mycobacteria are lipopolysaccharide (LPS) and lipoarabinomannans (LAMs), respectively. LAM isolated from pathogenic mycobacteria, such as Mycobacterium tuberculosis and M. bovis is capped with mannose residues at the nonreducing arabinofuranosyl termini (ManLAM), whereas LAM from rapidly growing a virulent mycobacteria lacks mannose caps (AraLAM) [5].

Alveolar macrophages harvested from patients with pulmonary infections, such as tuberculosis [6], release TNF- $\alpha$ spontaneously in culture. However, there has not as yet been a demonstration of TNF- $\alpha$ release by human alveolar macrophages from healthy subjects in response to these mycobacterial preparations, showing that the release of TNF- $\alpha$ by alveolar macrophages in response to purified LAMs would be beneficial in understanding the pathogenesis of mycobacterial diseases.

The aims of this study were two-fold. First, the intention was to test the stimulatory capacity of purified mycobacterial preparations in human alveolar macrophage cultures. The inclusion of LPS in the study served as a positive control, since LPS has been shown to be a potent stimulator of alveolar macrophages to produce TNF- $\alpha$. Secondly, the ability of inhaled preparations of corticosteroid to inhibit TNF- $\alpha$ release from alveolar macrophage populations, cultured ex vivo, was investigated. One of the inhaled corticosteroid preparations under test was a new chlorofluorocarbon (CFC)-free formulation of beclomethasone dipropionate (BDP), which utilized the propellant hydrofluoroalkane 134a (HFA) (QVAR ${ }^{\mathrm{TM}}$ (3M Pharmaceuticals, St Paul, MN, USA), HFA-BDP). HFA-BDP has been shown to have a finer particle size distribution, resulting in greater alveolar deposition than obtained using current CFC-BDP products [7]. It was predicted that HFA-BDP would show greater inhibition of TNF- $\alpha$ release and higher bronchoalveolar lavage fluid levels than would CFC-BDP. 


\section{Subjects and methods}

\section{Study patients and subjects}

Six patients with inflammatory pulmonary diseases, who were undergoing routine diagnostic bronchoscopic examination, were included in a preliminary study. These patients were aged 34-72 yrs. Twenty-eight healthy nonsmoking volunteers, aged 21-42 yrs, were then studied. The project was approved by the St. Mary's Hospital Local Research Ethics Committee.

Bronchoalveolar lavage. Fibreoptic bronchoscopy and bronchoalveolar lavage (BAL) were performed by the same operator on the control subjects with their full informed consent, using an Olympus BF P20D fibreoptic bronchoscope (Keymed, Southend, UK). Subjects were premedicated using midazolam and fentanyl given intravenously. Topical lignocaine was applied to the vocal cords, trachea and right main bronchus. The bronchoscope tip was wedged in a segmental orifice of the right middle lobe and five $50-\mathrm{mL}$ aliquots of sterile saline instilled in an identical fashion in each volunteer. At least $60 \%$ of this fluid was reaspirated and collected in a 500-mL polypropylene bottle, placed on ice and immediately transferred for ex vivo culture. Each subject underwent two fibreoptic bronchoscopic 14 days apart at either end of the course of inhaled medication. The second procedure was carried out 90-120 min after the last dose of inhaled medication was taken.

Preparation of alveolar macrophages. Alveolar macrophages were separated and cultured as described previously [8]. Briefly, cells were counted, centrifuged $(600 \times g, 10$ $\mathrm{min})$ at room temperature $\left(20^{\circ} \mathrm{C}\right)$ and washed once in phosphate-buffered saline before resuspension in RPMI 1640 medium supplemented with $5 \mathrm{mM} \mathrm{N}$-2-hydroxyethylpiperazine- $N-2$ ethanesulphonic acid HEPES, $10 \%$ foetal calf serum, $2 \mathrm{mM}$ glutamine and $100 \mathrm{UmL}^{-1}$ penicillin and 100 $\mu \mathrm{M}$ streptomycin at a density of $10^{6}$ cells $\cdot$ well $^{-1}$. The viability of the alveolar macrophages was determined in representative samples by means of trypan blue exclusion. After $1 \mathrm{~h}$ of incubation, nonadherent cells were washed off and the adherent population resuspended in $1 \mathrm{~mL}$ of medium containing either $10 \mu \mathrm{g} \cdot \mathrm{mL}^{-1}$ LPS Escherichia coli 026:B6, Sigma Chemical Co., Dorset, UK), $10 \mu \mathrm{g} \cdot \mathrm{mL}^{-1}$ AraLAM, $10 \mu \mathrm{g} \cdot \mathrm{mL}^{-1}$ ManLAM or medium alone in separate tissue culture dishes for $24 \mathrm{~h}$. ManLAM isolated from M. tuberculosis strain H37Rv and AraLAM from a rapid growing Mycobacterium species were provided by J. Belisle (Colorado State University). Endotoxin contamination in these preparations was $1.38 \mathrm{ng} \cdot \mathrm{mg}^{-1}$ for both ManLAM and AraLAM, as determined by the Limulus amoebocyte Assay (personal communication, J. Belisle). The adherent population of cells was found to be $90-95 \%$ alveolar macrophages, by both cytological examination and immunofluorescent staining using antihuman CD68 (Dako, High Wycombe, UK).

Measurement of beclomethasone 17-monopropionate concentration in bronchoalveolar lavage fluid supernatants. Supernatants from centrifuged unstimulated bronchoalveolar lavage (BAL) specimens from each volunteer were stored at $-70^{\circ} \mathrm{C}$ and tested in one batch. After solid-phase extraction, BAL fluid concentrations of beclomethasone 17monophosphate (17-BMP) were measured using a liquid chromatography/mass spectrometry method developed at 3M Pharmaceuticals. This method was automated with the MultiProbe (Packard, Chicago, IL, USA) using the Empore C1896-wells format (3M Pharmaceuticals) for extraction. The linear range was $75-2000 \mathrm{pg} \cdot \mathrm{mL}^{-1}$ for $17-\mathrm{BMP}$ in BAL. The lavage concentrations were calculated using standard curves that were fitted to a weighted linear regression with correlations ranging 0.9958-0.9997. The signal-to-noise ratio at the limit of quantification was $>10: 1$.

Measurement of tumour necrosis factor- $\alpha$ bioactivity. The biological activity of TNF- $\alpha$ in the culture supernatants was measured in triplicate, as previously described [9], using murine L929 cells to assay for cytotoxicity in the presence of actinomycin D. Confirmation of the TNF- $\alpha$ concentration in selected samples was obtained using an enzyme-linked immunosorbent assay kit (Human TNF- $\alpha$ Quantikine; R\&D Systems, Abingdon, UK) (results not shown).

Inhaled steroid regime. Following the first bronchoscopic examination, control subjects were started on a course of either inhaled BDP or placebo, administered via a pressand-breathe metered dose inhaler, randomized in an openlabelled manner. BDP was given at a dose of $400 \mu \mathrm{g}$ (four puffs) twice daily as either HFA-BDP (QVAR $\left.{ }^{\mathrm{TM}}\right)$ or CFCBDP (FILAIR ${ }^{\mathrm{TM}}$, 3M Pharmaceuticals). The placebo was an aerosolized preparation using the HFA propellant (HFAplacebo). Subjects on placebo followed the same protocol as those on the inhaled steroid regimen (i.e. four puffs taken morning and evening). Inhaler technique was checked and monitored, and compliance was supervised by an experienced asthma nurse.

Statistical analysis. Since the raw data included at least triplicate values for each culture supernatant and standard curve sample, a statistical test was used which took advantage of both the mean and standard error of the mean for each data point. In order to test for differences in TNF- $\alpha$ levels before and after the two active BDP treatments compared to placebo, a linear model allowing for fixed patient effects was fitted to the logarithms of the TNF- $\alpha$ concentrations. A fixed effects term for the three ex vivo treatments was also included. As the TNF- $\alpha$ bioassay reports only mean and standard error of the three measured TNF- $\alpha$ concentrations, it was necessary to simulate three data points having the appropriate mean and standard error for each subject's TNF- $\alpha$ concentrations. The results show the change in log TNF- $\alpha$ concentration post-inhaler compared to placebo for each of the three ex vivo treatments. A pvalue of $<0.05$ was considered significant. The analysis was performed by fitting linear models in Splus (Cambridge, MA, USA), each of the three drug groups, HFA-placebo, HFA-BDP and CFC-BDP, being fitted simultaneously to the model. The BAL fluid 17-BMP concentration data were analysed using Fisher's exact test.

\section{Results}

Production of tumour necrosis factor- $\alpha$ by alveolar macrophages in response to lipopolysaccharide and lipoarabinomannan

Preliminary experiments on alveolar macrophages from six hospital patients undergoing diagnostic bronchoscopy were performed to characterize the titration of LPS and 
Table 1. - Tumour necrosis factor- $\alpha$ (TNF- $\alpha$ ) concentrations from initial titration experiments to characterize the optimal concentrations of glycolipid preparation and duration of stimulation required

\begin{tabular}{lcc}
\hline & \multicolumn{2}{c}{ TNF- $\alpha \mathrm{ng} \cdot \mathrm{mL}^{-1}$} \\
\cline { 2 - 3 } Ex vivo stimulant & 6-h culture & 24-h culture \\
\hline Medium alone & $5.16 \pm 1.68$ & $2.02 \pm 0.98$ \\
LPS 10 $\mu \mathrm{g} \cdot \mathrm{mL}^{-1}$ & $>12$ & $>12$ \\
AraLAM 1 $\mu \mathrm{g} \cdot \mathrm{mL}^{-1}$ & $5.66 \pm 2.59$ & $2.87 \pm 2.23$ \\
AraLAM 3 $\mu \mathrm{g} \cdot \mathrm{mL}^{-1}$ & $6.65 \pm 3.04$ & $3.47 \pm 2.92$ \\
AraLAM 10 $\mu \mathrm{g} \cdot \mathrm{mL}^{-1}$ & $7.74 \pm 3.38$ & $8.96 \pm 7.54$ \\
ManLAM 1 $\mu \mathrm{g} \cdot \mathrm{mL}^{-1}$ & $4.83 \pm 2.63$ & $2.30 \pm 1.81$ \\
ManLAM 3 $\mu \mathrm{g} \cdot \mathrm{mL}^{-1}$ & $5.28 \pm 2.75$ & $3.11 \pm 2.41$ \\
ManLAM 10 $\mu \mathrm{g} \cdot \mathrm{mL}^{-1}$ & $6.16 \pm 2.87$ & $8.88 \pm 2.83$ \\
\hline
\end{tabular}

Data are presented as mean \pm SEM. TNF- $\alpha$ concentrations were measured by means of enzyme-linked immunosorbent assay of the alveolar macrophage culture supernatants from the bronchoalveolar lavage specimens of six patients. LPS: lipopolysaccharide; AraLAM: arabinofuranosyl lipoarabinomannan (LAM); ManLAM: mannose LAM.

LAM and also the optimal length of time of cell culture. Results indicated that a 24-h culture time resulted in the induction of maximal TNF- $\alpha$ bioactivity by LPS and both LAM preparations at a concentration of $10 \mu \mathrm{g} \cdot \mathrm{mL}^{-1}$. Not surprisingly, alveolar macrophages incubated with lower concentrations of LAMs produced lower but statistically nonsignificant concentrations of TNF- $\alpha$ (table 1).

The geometric mean and 95\% confidence interval of TNF- $\alpha$ concentrations in alveolar macrophage culture supernatants were calculated for the group of 28 control subjects, for the pre-inhaler 24-h ex vivo cultures (table 2). In the combined pretreatment groups, TNF- $\alpha$ concentrations in alveolar macrophage culture supernatants were significantly higher when culture was performed in the presence of LPS $(p<0.001)$ and in the presence of AraLAM $(p<0.001)$ (fig. 1a and $c)$. The difference was less marked, although still significant, when culture was performed in the presence of ManLAM ( $\mathrm{p}=0.02)$ compared to medium alone (fig. 1b).

Beclomethasone 17-monopropionate concentrations in cell-free bronchoalveolar lavage supernatants

No measurable 17-BMP was detectable in any of the pretreatment BAL supernatants or in any of the posttreatment BAL fluid supernatants from the HFA-placebo

Table 2. - Tumour necrosis factor- $\alpha$ (TNF- $\alpha$ ) concentrations in alveolar macrophage culture supernatants stimulated with one of the three glycolipids, lipopolysaccharide (LPS), arabinofuranosyl lipoarabinomannan (LAM) (AraLAM) and mannose LAM (ManLAM), from 28 healthy volunteers*

\begin{tabular}{lccc}
\hline Ex vivo stimulant & $\begin{array}{c}\mathrm{TNF}-\alpha \\
\mathrm{ng} \cdot \mathrm{mL}^{-1}\end{array}$ & $\begin{array}{c}95 \% \text { confidence } \\
\text { interval }\end{array}$ & p-value \\
\hline Medium alone & 0.72 & $0.48-1.07$ & \\
LPS $10 \mu \mathrm{g} \cdot \mathrm{mL}^{-1}$ & 138 & $110-173$ & $<0.001$ \\
ManLAM $10 \mu \mathrm{g} \cdot \mathrm{mL}^{-1}$ & 1.42 & $0.94-2.14$ & 0.02 \\
AraLAM $10 \mu \mathrm{g} \cdot \mathrm{mL}^{-1}$ & 12.6 & $8.9-17.7$ & $<0.001$ \\
\hline
\end{tabular}

*: taken from placebo, chlorofluorocarbon and hydrofluoroalkane groups prior to any inhaled steroid preparation. Data are presented as geometric mean. group. There was marked variability in the post-treatment values from the HFA-BDP and CFC-BDP groups (table 3 ). However, seven of the 10 post-treatment BAL fluid supernatants harvested from subjects who were randomized to CFC showed measurable BAL fluid 17-BMP concentrations ranging $77.4-3165 \mathrm{pg} \cdot \mathrm{mL}^{-1}$. Only one of 10 post-treatment BAL fluid supernatants from the HFABDP group had a measurable BAL fluid concentration. There was a significantly greater chance of the 17-BMP concentration being higher in the CFC-BDP group than in the HFA-BDP group. This reached statistical significance $(\mathrm{p}=0.02)$.

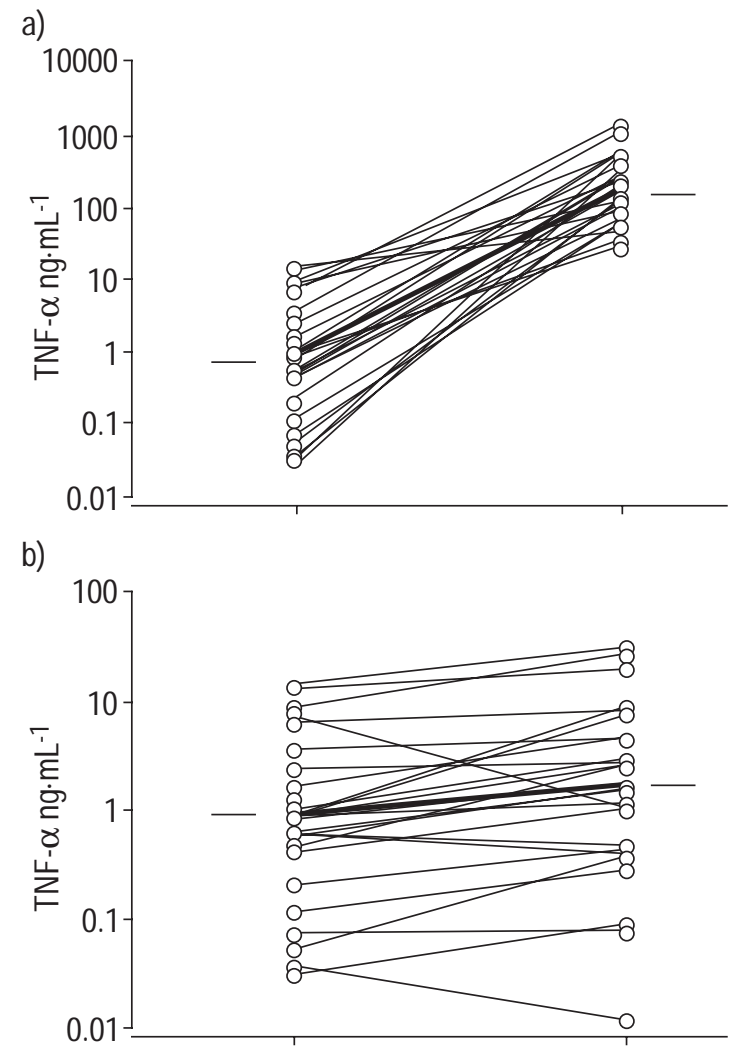

c)

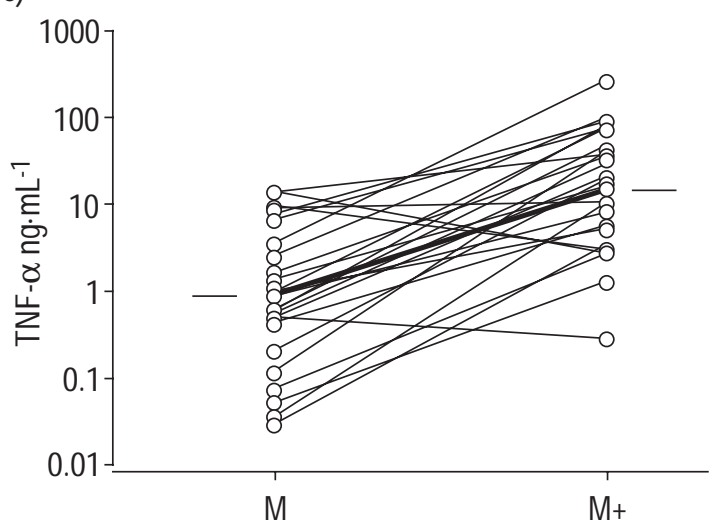

Fig. 1. - Tumour necrosis factor (TNF- $\alpha$ ) bioactivity in 24-h culture supernatants of alveolar macrophages from healthy nonsmoking volunteers incubated with: a) lipopolysaccharide $\left(10 \mu \mathrm{g} \cdot \mathrm{mL}^{-1}\right)(\mathrm{p}<0.001)$; b) mannose lipoarabinomannan (LAM) $\left(10 \mu \mathrm{g} \cdot \mathrm{mL}^{-1}\right)$; and c) arabirofuranosyl LAM $\left(10 \mu \mathrm{g} \cdot \mathrm{mL}^{-1}\right)\left(\mathrm{M}^{+}\right)(\mathrm{p}<0.01)$ compared to culture supernatants from unstimulated alveolar macrophages (medium alone (M)). The horizontal bars and thick lines represent the geometric mean. 
Table 3. - Beclomethasone 17-monopropionate(17-BMP) concentrations in bronchoalveolar lavage fluid supernatants from healthy volunteers following a 14-day course of either inhaled chlorofluorocarbon (CFC)-beclomethasone diproprionate (BDP) or hydrofluoroalkane 134a (HFA)$\mathrm{BDP}$ at a dose of $800 \mu \mathrm{g} \cdot \mathrm{day}^{-1 *}$

\begin{tabular}{|c|c|c|}
\hline \multirow[b]{2}{*}{ Volunteer No. } & \multicolumn{2}{|c|}{ 17-BMP pg.mL ${ }^{-1}$} \\
\hline & HFA-BDP & CFC-BDP \\
\hline $1 / 11$ & $<75$ & $<75$ \\
\hline $2 / 12$ & $<75$ & 86.3 \\
\hline $3 / 13$ & $<75$ & $<75$ \\
\hline $4 / 14$ & $<75$ & 76.1 \\
\hline $5 / 15$ & $<75$ & 100.6 \\
\hline $6 / 16$ & $<75$ & 80.6 \\
\hline $7 / 17$ & $<75$ & 452.3 \\
\hline $8 / 18$ & $<75$ & 3165 \\
\hline 9/19 & 122.8 & 77.4 \\
\hline $10 / 20$ & $<75$ & $<75$ \\
\hline
\end{tabular}

*: these results correspond to the same groups of volunteers as used in figures 2 and 3.

Tumour necrosis factor- $\alpha$ production by stimulated cultured alveolar macrophages following in vivo administration of inhaled hydrofluoroalkane 134a-beclomethasone dipropionate preparation

Administration of HFA-BDP $\left(800 \mu \mathrm{g} \cdot \mathrm{day}^{-1}\right)$ resulted in a significant reduction in TNF- $\alpha$ production in the group of 10 subjects randomized to inhaled HFA-BDP. Log TNF- $\alpha$ concentrations in culture supernatants of LPS-stimulated
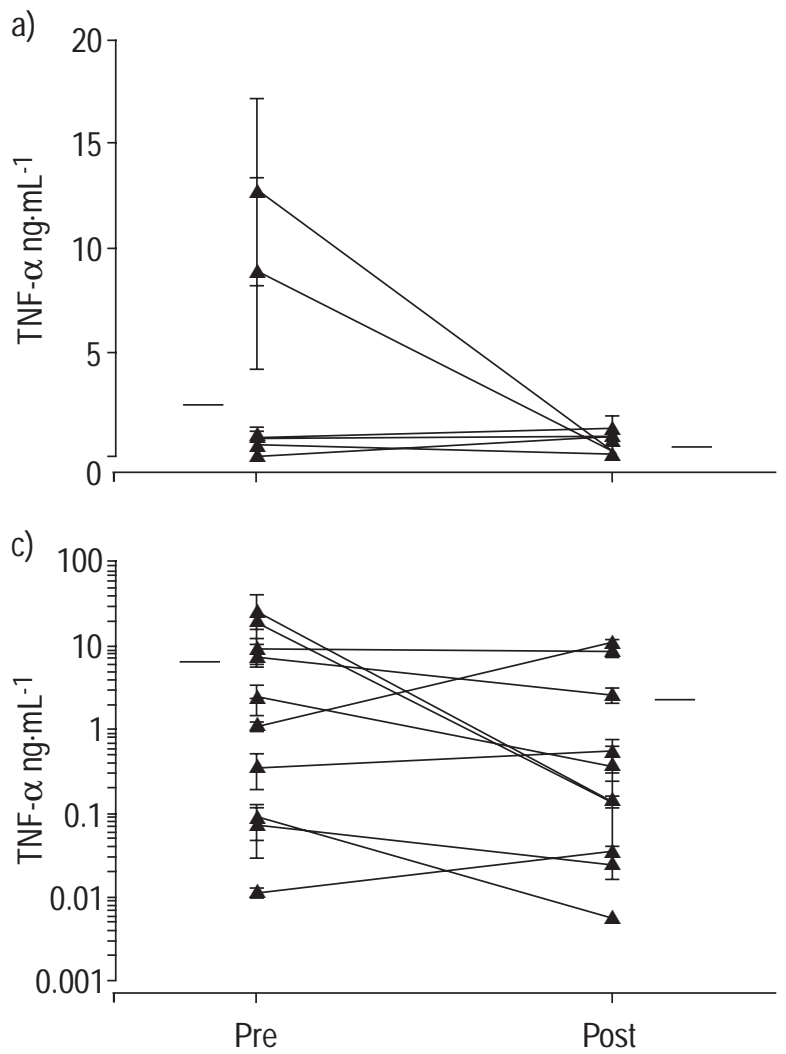

Fig. 2 - Tumour necrosis factor- $\alpha$ (TNF- $\alpha$ ) bioactivity of alveolar macrophage culture supernatants from the inhaled hydrofluoroalkane 134 abeclomethasone dipropionate volunteer group stimulated with: a) medium alone (Ns); b) lipopolysaccharide $\left(10 \mu \mathrm{g} \cdot \mathrm{mL}^{-1}\right)(\mathrm{p}<0.001)$; $\left.\mathrm{c}\right) \mathrm{mannose}^{-}$ lipoarabinomannan (LAM) $\left(10 \mu \mathrm{g} \cdot \mathrm{mL}^{-1}\right)(\mathrm{p}=0.02)$; and $\left.\mathrm{d}\right)$ arabinofuranosyl LAM $\left(10 \mu \mathrm{g} \cdot \mathrm{mL}^{-1}\right)(\mathrm{NS})$. Each symbol corresponds to one volunteer. The horizontal bars represent the geometric mean. Pre: pre-steroid; Post: post-steroid.

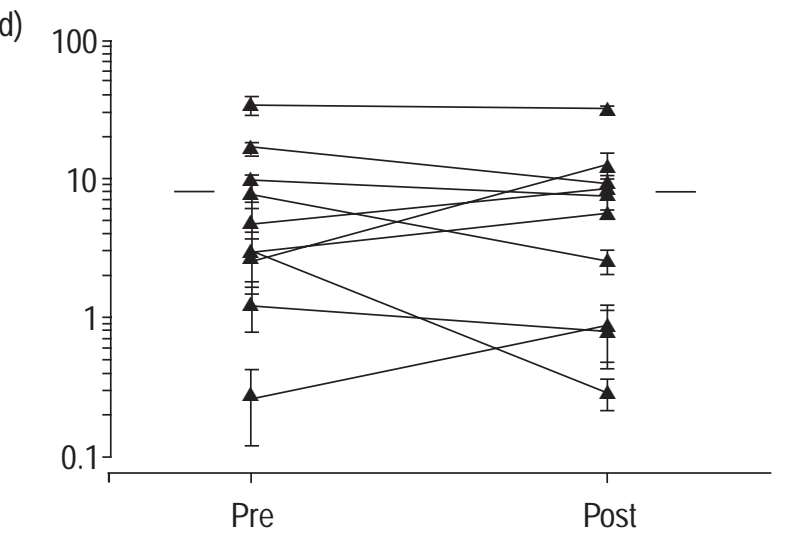

and ManLAM-stimulated alveolar macrophages decreased significantly compared to those in the group taking inhaled placebo ( $\mathrm{p}<0.001$ and $\mathrm{p}<0.02$ respectively) (fig. $2 \mathrm{~b}$ and $\mathrm{c}$ ). However, the decrease in mean alveolar macrophage culture supernatant TNF- $\alpha$ concentrations following AraLAM stimulation did not reach significance (fig. 2d). TNF- $\alpha$ levels in unstimulated alveolar macrophage culture supernatants from this group did not fall significantly following 2 weeks of inhaled HFA-BDP (fig. 2a).

Tumour necrosis factor- $\alpha$ production by stimulated cultured alveolar macrophages following in vivo administration of inhaled chlorofluorocarbon-beclomethasone dipropionate preparation

There was no statistically significant change in TNF- $\alpha$ production by alveolar macrophages stimulated with LPS, ManLAM, or AraLAM from the 10 patients studied prior to and following 14 days' treatment with inhaled CFC-BDP at a dose of $800 \mu \mathrm{g} \cdot \mathrm{day}^{-1}$, compared to placebo (fig. 3).

Tumour necrosis factor- $\alpha$ production by stimulated cultured alveolar macrophages following in vivo administration of inhaled hydrofluoroalkane134a-placebo preparation

In the group of eight subjects randomized to the inhaled placebo preparation, there was no significant change in $\log$ TNF- $\alpha$ concentration pre- and post-inhaler in LPS-, AraLAM- and ManLAM-stimulated alveolar macrophage culture supernatants. When supernatants of cells cultured

b)

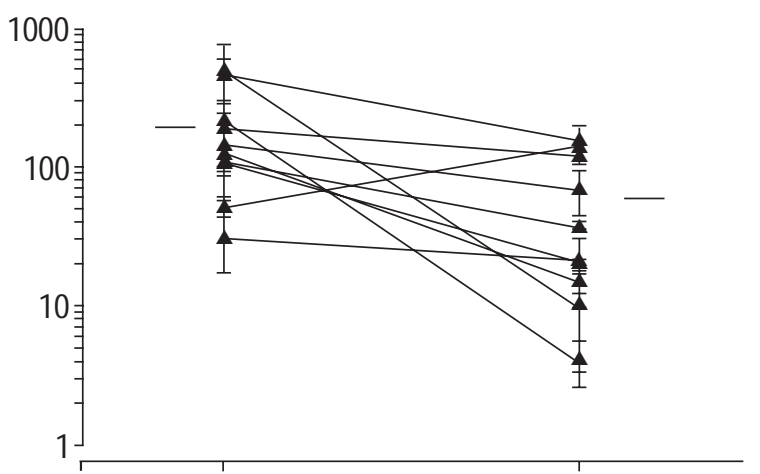




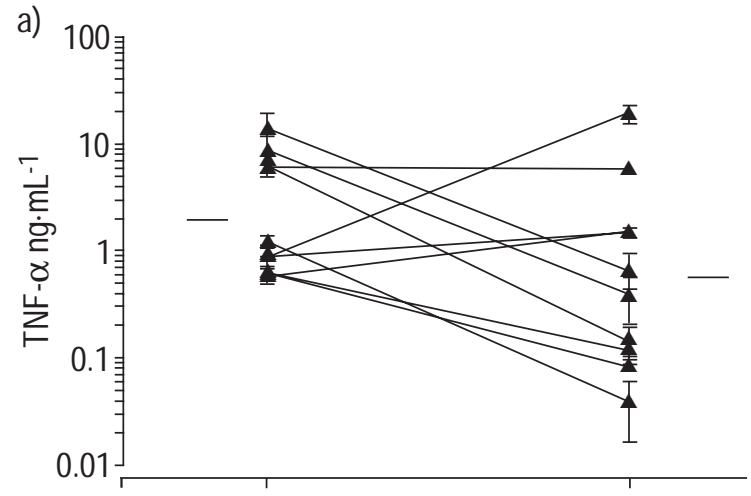

b)

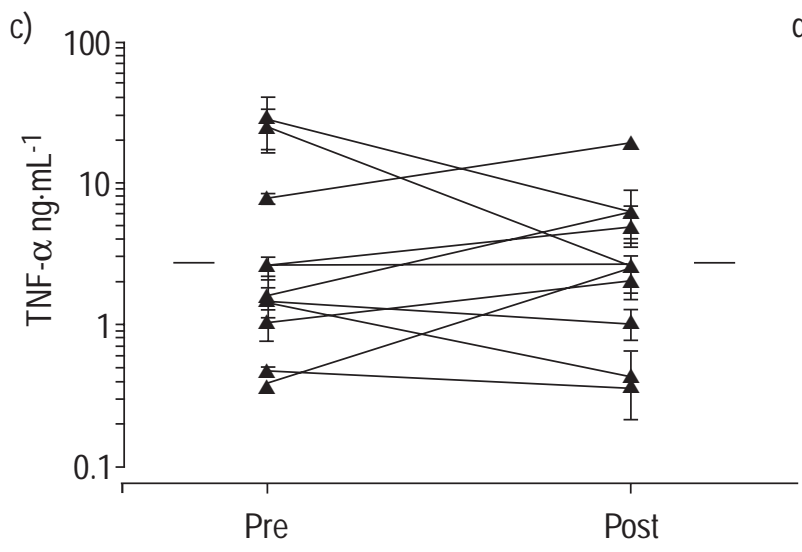

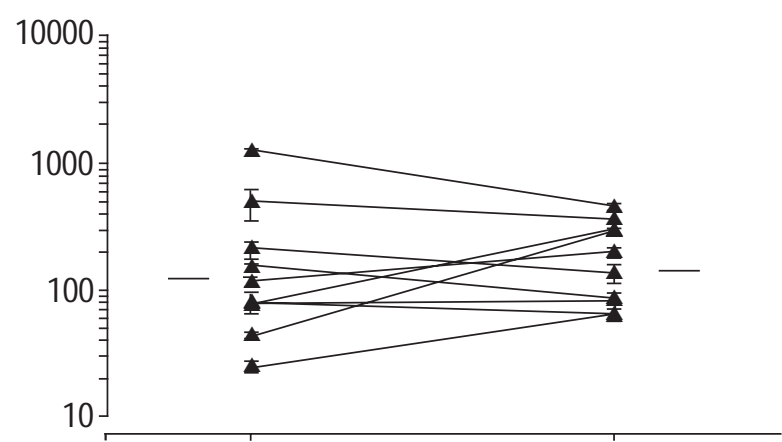

d)

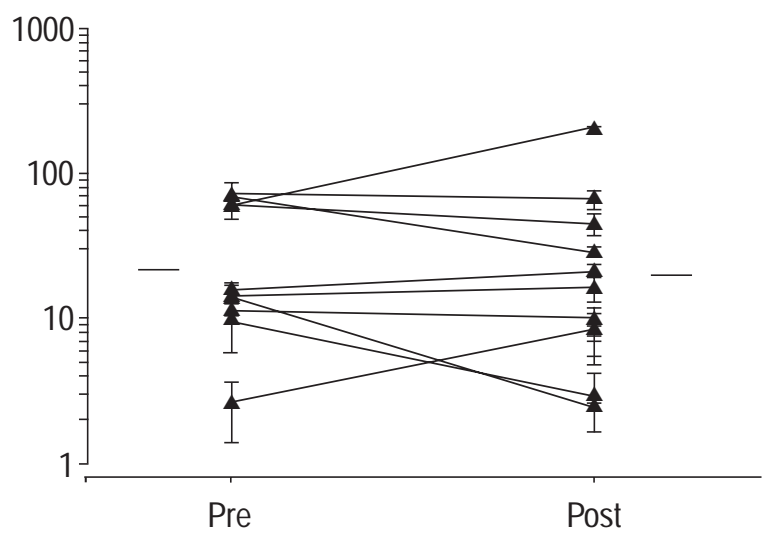

Fig. 3. - Tumour necrosis factor- $\alpha$ (TNF- $\alpha$ ) bioactivity of alveolar macrophage culture supernatants from the inhaled chlorofluorocarbon-beclomethasone dipropionate volunteers stimulated with: a) medium alone (Ns); b) lipopolysaccharide $\left(10 \mu \mathrm{g} \cdot \mathrm{mL}^{-1}\right)(\mathrm{Ns})$; c) mannose lipoarabinomannan (LAM) $\left(10 \mu \mathrm{g} \cdot \mathrm{mL}^{-1}\right)(\mathrm{Ns})$; and d) arabinofuranosyl LAM $\left(10 \mu \mathrm{g} \cdot \mathrm{mL}^{-1}\right)$ (Ns). Each symbol corresponds to one volunteer (11-20). The horizontal bars represent the geometric mean. Pre: pre-steroid; Post: post-steroid.

in medium alone collected prior to and following a 2-week course of placebo inhaler were compared, there was a small but statistically significant reduction $(\mathrm{p}=0.04)$ in TNF- $\alpha$ release (fig. 4 ).

\section{Discussion}

In this study, the first clear evidence is provided that a novel inhaled CFC-free preparation of BDP (HFA-BDP), with increased peripheral lung deposition, can modulate glycolipid-induced TNF- $\alpha$ production by human alveolar macrophages, in comparison with CFC-BDP and HFAplacebo. A dose of $400 \mu \mathrm{g}$ HFA-BDP, administered twice daily for 2 weeks, significantly reduced glycolipid-induced TNF- $\alpha$ production by alveolar macrophages cultured $e x$ vivo. However, following the administration of CFC-BDP or HFA-placebo at an identical dose, no change in TNF- $\alpha$ production in response to the same preparations was observed. As physiological and pathological evidence suggests that the entire pulmonary airway, including peripheral elements and lung parenchyma, is involved in the inflammatory processes in asthma $[10,11]$, these findings imply that inhaled corticosteroid drug distribution to the alveoli might be beneficial for the treatment of asthma.

This report provides the first evidence that two preparations of LAM, AraLAM and ManLAM, the major cell wall glycolipids of mycobacteria, are able to elicit TNF- $\alpha$ production from human alveolar macrophages obtained from healthy human volunteers. This study indicates that ManLAM from virulent $M$. tuberculosis has a lesser capacity to activate human alveolar macrophages to produce TNF- $\alpha$ than the structurally similar AraLAM, purified from rapidly growing nonpathogenic mycobacteria. These data corroborate two recent studies which have demonstrated that live preparations of two laboratory strains of $M$. tuberculosis induce TNF- $\alpha$ production in primary human alveolar macrophages $[12,13]$. The results are also consistent with the differential responses to mycobacterial LAMs of human and murine mononuclear phagocytes, reported by several other investigators [1418]. The differences in cytokine induction by the two preparations has been attributed to variations in the nonreducing termini of the two molecules $[5,19]$. The results in this human volunteer study, however, showed more interindividual variation in the ability of ManLAM to induce TNF- $\alpha$ production in alveolar macrophages, compared to AraLAM (fig. $1 \mathrm{~b} \mathrm{c}$ ). Overall, the mean increase in TNF- $\alpha$ concentration in supernatants of AraLAMstimulated alveolar macrophages was highly significant $(\mathrm{p}<0.001)$ when compared to the TNF- $\alpha$ bioactivity from unstimulated alveolar macrophage cultures; the increase was less marked in supernatants of ManLAM-stimulated cells $(\mathrm{p}=0.02)$. The TNF- $\alpha$ release induced by Gram-negative bacterial LPS in the present study was substantial and an order of magnitude greater than that from AraLAMinduced alveolar macrophages. This finding of LPSinduced TNF- $\alpha$ production was similar to that in previous studies $[12,20,21]$. 


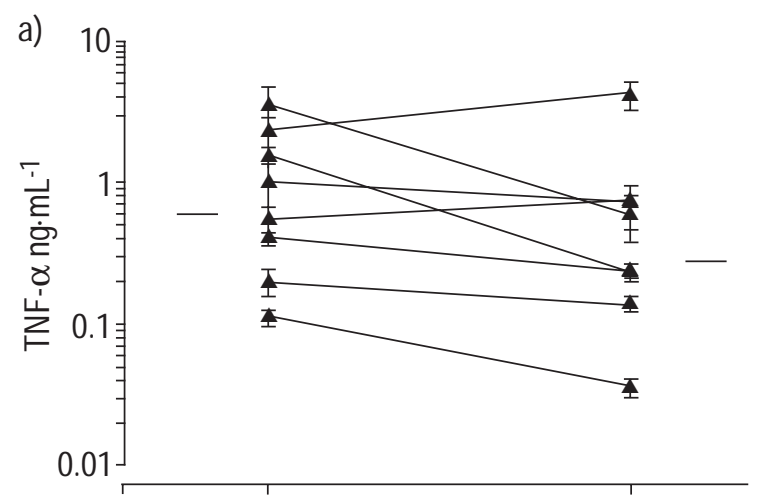

b)
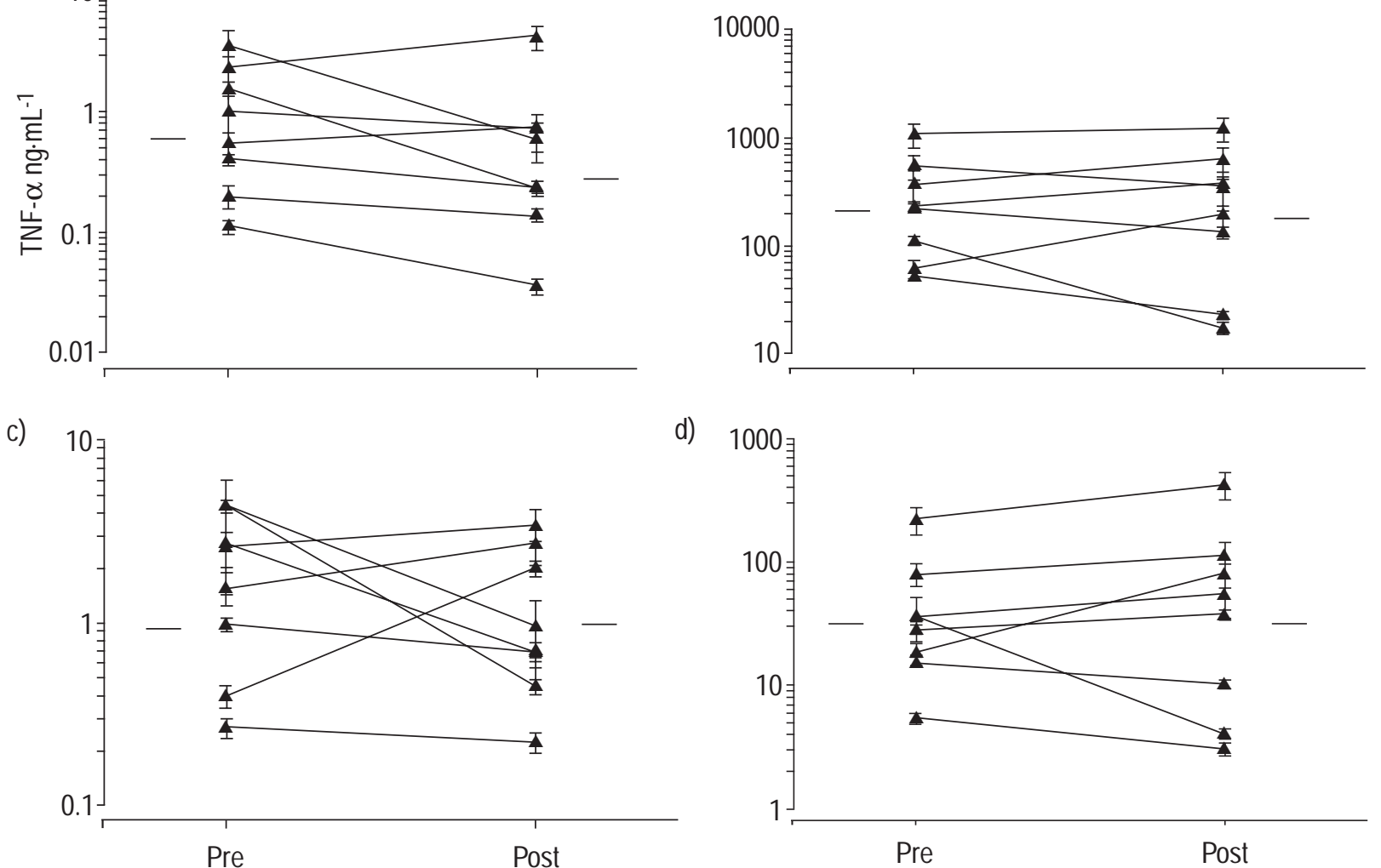

Fig. 4. - Tumour necrosis factor- $\alpha$ (TNF- $\alpha)$ bioactivity of alveolar macrophage culture supernatants from the hydrofluoroalkane-placebo volunteer group stimulated with: a) medium alone $(\mathrm{p}=0.04)$; b) lipopolysaccharide $\left.\left(10 \mu \mathrm{g} \cdot \mathrm{mL}^{-1}\right)(\mathrm{NS}) ; \mathrm{c}\right)$ mannose lipoarabinomannan $(\mathrm{LAM})\left(10 \mu \mathrm{g} \cdot \mathrm{mL}^{-1}\right)(\mathrm{NS})$; and d) arabinofuranosyl LAM $\left(10 \mu \mathrm{g} \cdot \mathrm{mL}^{-1}\right)$ (Ns). Each symbol corresponds to one volunteer (21-28). The horizontal bars represent the geometric mean. Pre: pre-placebo; Post: post-placebo.

It was intriguing that TNF- $\alpha$ production by AraLAMstimulated alveolar macrophages was not significantly modulated $(p=0.2)$ by a course of HFA-BDP. Since AraLAM binds to the same high-affinity CD14 macrophage receptor as LPS [22], leading to transduction of an activation signal for TNF- $\alpha$ production [21], it might be expected that corticosteroids would modulate the effects of AraLAM and LPS similarly, as has been demonstrated previously [23]. However, the signal transduction pathway initiated by AraLAM binding to CD14 is not well characterized [24], and recent studies have demonstrated that, although LPS and AraLAM share some CD14-dependent responses, the signal transduction pathway for AraLAM requires one or more components that are at least partially distinct from that initiated by LPS $[16,25]$, raising the possibility of different regulatory steps. By contrast, ManLAM is able to signal through a different receptor, the macrophage mannose receptor [26], and thus might also be subject to differing mechanisms of immunomodulation by corticosteroid. This might explain the significant modulatory effect of BDP on TNF- $\alpha$ induction by ManLAMstimulated alveolar macrophages.

The results of the 17-BMP concentration measurements in BAL fluid supernatants from volunteers were unexpected. Although an in vivo course of inhaled CFC-BDP had no significant modulatory effect on alveolar macrophage function, BAL fluid levels of its active metabolite, 17-BMP, were significantly increased compared to 17 -
BMP levels following HFA-BDP. A key to understanding this observation can be found in the substantial particle size differences between CFC-BDP and HFA-BDP [7]. Whereas CFC-BDP delivers an aerosol with particles of mass medium aerodynamic diameter (MMAD) of 3.5-4.0 $\mu \mathrm{m}$, HFA-BDP delivers particles with a MMAD of $1.1 \mu \mathrm{m}$. The present findings suggest that the smaller-sized particles from HFA-BDP result in greater lung distribution and greater alveolar uptake, which in turn results in a higher drug concentration at the receptor site than obtained with the larger drug particles from CFC-BDP. The high concentrations of 17-BMP in BAL fluid resulting from CFC-BDP administration probably reflects the poorer drug distribution in the lungs and the reduced absorption of the large particles. It is interesting to note that BAL fluid levels appear to measure drug which is not absorbed and presumably not efficacious.

In summary, this study demonstrates that human alveolar macrophages from healthy volunteers activated $e x$ vivo by glycolipids of Gram-negative bacteria and mycobacteria respond by upregulating tumour necrosis factor- $\alpha$ release. This inflammatory response can be modulated by the peripheral deposition of inhaled corticosteroid in vivo. It was observed that inhaled corticosteroids must reach the distal airways to be effective. These findings may have important implications in the development of new immunotherapeutic agents designed to improve treatment of inflammatory pulmonary disorders. 
Acknowledgements. The authors thank J.Y. Belisle, Colorado State University, College of Veterinary Medicine and Biomedical Sciences, Fort Collins, CO, USA for supplying purified lipoarabinomannan preparations (National Institutes of Health/National Institute of Allergy and Infectious Diseases contract AI 25147 to Colorado State University). The authors appreciate the assistance of R. Arnold, Dept of Epidemiology and Public Health, Imperial College School of Medicine, St. Mary's Campus, London for statistical analysis, and C. Wagner and S. Kurup for assistance with the bronchoalveolar lavage fluid drug assays.

\section{References}

1. Cembrzynska-Nowak M, Szklarz E, Inglot AD, Teodorczyk-Injeya JA. Elevated release of tumour necrosis factor- $\alpha$ and interferon- $\gamma$ by bronchalveolar leukocytes from patients with bronchial asthma. Am Rev Respir Dis 1993; 147: 291-295.

2. Piguet PF, Collart MA, Grau GE, Sappino AE, Vassalli P. Requirement of tumour necrosis factor for development of silica-induced pulmonary fibrosis. Nature 1990; 344 : 245-247.

3. Flynn J, Goldstein M, Chan J, et al. Tumour necrosis factor-alpha is required in the protective immune response against Mycobacterium tuberculosis in mice. Immunity 1995; 2: 561-572.

4. Rook GAW, Taverne J, Moreno C. The role of tumour necrosis factor in the pathogenesis of tuberculosis and leprosy, and the relationship between tissue-damaging pathology and production of agalactosyl IgG. In: Bonavida B, Granger G, eds. Tumour Necrosis Factor: Structure, Mechanism of Action, Role in Disease and Therapy. Basle, Karger, 1990; pp. 168-176.

5. Chatterjee D, Roberts AD, Rivoire B, McNeil M, Brennan PJ. Lipoarabinomannan of Mycobacterium tuberculosis. Capping with mannosyl residues in some strains. $J$ Biol Chem 1992; 267: 6234-6239.

6. Law K, Weiden M, Harkin T, Tchou-Wong K, Chi C, Rom WN. Increased release of interleukin- $1 \beta$ interleukin6 and tumour necrosis factor- $\alpha$ by bronchoalveolar cells lavaged from involved sites in pulmonary tuberculosis. Am J Respir Crit Care Med 1996; 153: 799-804.

7. Leach C. Enhanced drug delivery through reformulating MDIs with HFA propellants: drug deposition and its effects on preclinical and clinical programmes. In: Dalby RN, Byron PR, Farr SJ, eds. Respiratory Drug Delivery V Proceedings. Buffalo Grove, Interpharm Press, 1996; pp. 133-144.

8. Haynes AR, Shaw RJ. Dexamethasone-induced increase in platelet-derived growth factor (B) mRNA in human alveolar macrophages and myelomonocytic HL60 macrophage-like cells. Am J Respir Cell Mol Biol 1992; 7: 198206.

9. Marshall BG, Chambers MA, Wangoo A, Shaw RJ, Young DB. Production of tumour necrosis factor and nitric oxide by macrophages infected with live and dead mycobacteria and their suppression by an interleukin-10secreting recombinant. Infect Immun 1997; 65: 1931-1935.

10. Kraft M, Djukanovic R, Wilson S, Holgate ST, Martin RJ. Alveolar tissue inflammation in asthma. Am J Respir Crit Care Med 1996; 154: 1505-1510.

11. Hamid Q, Song Y, Kotsimbos TC, et al. Respiratory pathophysiologic responses: inflammation of small airways in asthma. J Allergy Clin Immunol 1997; 100: 44-51.

12. Fenton MJ, Vermeulen MW, Kim S, Burdick M, Strieter $\mathrm{RM}$, Kornfeld H. Induction of interferon-gamma production in human alveolar macrophages by Mycobacterium tuberculosis. Infect Immun 1996; 5: 5149-5156.

13. Keane J, Balcewicz-Sablinska MK, Remold HG, et al. Infection by Mycobacterium tuberculosis promotes human alveolar macrophage apoptosis. Infect Immun 1997; 65: 298-304.

14. Moreno C, Taverne J, Mehlert A, et al. Lipoarabinomannan from Mycobacterium tuberculosis induces the production of tumour necrosis factor from human and murine macrophages. Clin Exp Immunol 1989; 76: 240245.

15. Dahl KE, Shiratsuchi H, Hamilton BD, Ellner JJ, Toossi $Z$. Selective induction of transforming growth factor beta in human monocytes by lipoarabinomannan of $\mathrm{Myco-}$ bacterium tuberculosis. Infect Immun 1996; 64: 399-405.

16. Bernardo J, Billingslea AM, Blumenthal RL, Seetoo KF, Simons ER, Fenton MJ. Differential responses of human mononuclear phagocytes to mycobacterial lipoarabinomannans: role of CD14 and the mannose receptor. Infect Immun 1998; 66: 28-35.

17. Adams LB, Fukutomi Y, Krahen JL. Regulation of murine macrophage effector functions by lipoarabinomannan from mycobacterial strains with different degrees of virulence. Infect Immun 1993; 61: 4173-4181.

18. Roach TI, Barton CH, Chatterjee D, Blackwell JM. Macrophage activation: lipoarabinomannan from avirulent and virulent strains of Mycobacterium tuberculosis differentially induces the early genes c-fos, $\mathrm{KC}, \mathrm{JE}$, and tumor necrosis factor-alpha. J Immunol 1993; 150: 18861896.

19. Barnes PF, Chatterjee D, Abrams JS, et al. Cytokine production induced by Mycobacterium tuberculosis lipoarabinomannan. Relationship to chemical structure. $J$ Immunol 1992; 149: 541-547.

20. Adams JL, Czuprynski CJ. Bacterial lipopolysaccharide induces release of tumor necrosis factor-alpha from bovine peripheral blood monocytes and alveolar macrophages in vitro. J Leukoc Biol 1990; 48: 549-556.

21. Dentener MA, Bazil V, Von Asmuth EJ, Ceska M, Buurman WA. Involvement of $\mathrm{CD} 14$ in lipopolysaccharide-induced tumor necrosis factor-alpha, IL-6 and IL-8 release by human monocytes and alveolar macrophages. J Immunol 1993; 150: 2885-2891.

22. Pugin J, Heumann A, Tomasz A, et al. CD14 is a pattern recognition receptor. Immunity 1994; 1: 509-516.

23. Han J, Thompson P, Beutler B. Dexamethasone and pentoxifylline inhibit endotoxin-induced cachectin/tumour necrosis factor synthesis at separate points in the signalling pathway. J Exp Med 1990; 172: 391-394.

24. Ernst JD. Macrophage receptors for Mycobacterium tuberculosis. Infect Immun 1998; 66: 1277-1281.

25. Savadra Jr. R, Delude RL, Ingalls RR, Fenton MJ, Golenbock DT. Mycobacterial lipoarabinomannan recognition requires a receptor that shares components with the endotoxin signalling system. J Immunol 1996; 157 : 2549-2554.

26. Schlesinger LS, Kaufman TM, Iyer S, Hull SR, Marchiando LK. Differences in mannose receptormediated uptake of lipoarabinomannan from virulent and attenuated strains of Mycobacterium tuberculosis. $J$ Immunol 1996; 157: 4568-4575. 